\title{
Diferencias en los valores de las dimensiones del burnout en educadores con y sin síntomas o problemas de salud
}

\section{Differences in the values of the Burnout Dimensions on Teachers with and Without Symptoms or Health Problems}

\author{
María S. Menghi \\ Pontifica Universidad Católica Argentina, Centro de Investigación Interdisciplinar en Valores, \\ Integración y Desarrollo Social, Paraná, Argentina \\ ORCID: https://orcid.org/0000-0002-6941-3482 \\ Lucas M. Rodriguez \\ Pontifica Universidad Católica Argentina, Centro de Investigación Interdisciplinar en Valores, \\ Integración y Desarrollo Social, Paraná, Argentina \\ ORCID: https://orcid.org/0000-0001-5525-1155

\section{María E. Oñate} \\ Pontifica Universidad Católica Argentina, Centro de Investigación Interdisciplinar en Valores, \\ Integración y Desarrollo Social, Paraná, Argentina \\ ORCID: https://orcid.org/0000-0003-3277-4011
}

Recibido 28-05-19 Revisado 30-06-19 Aprobado 13-09-19 En línea 18-09-19

*Correspondencia

Email: msmenghi@uca.edu.ar
Citar como:
Menghi, M. S., Rodriguez, L.M., \& Oñate, M. E. (2019). Diferencias en los valores de las dimensiones del burnout en educadores con y sin síntomas o problemas de salud. Propósitos y Representaciones, 7(3), 179-197. doi: http://dx.doi.org/10.20511/pyr2019.v7n3.338 


\section{Resumen}

El objetivo del presente estudio fue observar si existen diferencias en los valores de las dimensiones del burnout entre quienes han experimentado síntomas o problemas de salud en el último año y quienes no, en una muestra de 223 maestros que trabajan en instituciones educativas (nivel primario). Los participantes completaron el Maslach Burnout Inventory (MBI), en su versión para educadores y una encuesta ad hoc. Se realizó una prueba t para muestras independientes con la finalidad de observar si ambos grupos de docentes difieren significativamente en los valores obtenidos en las dimensiones del MBI (agotamiento emocional, despersonalización y falta de realización personal). La prueba t mostró diferencias significativas entre los grupos de educadores, precisamente en las dimensiones de agotamiento emocional y la despersonalización, con valores más altos en el grupo de maestros que manifestaron sufrir síntomas o problemas de salud en los últimos 12 meses.

Palabras clave: Docente de escuela primaria; Estrés laboral; Salud mental; Agotamiento emocional; Despersonalización.

\section{Summary}

The aim of the present study was to observe if there are differences in the level of the burnout dimensions between teachers who have experienced symptoms or health problems in the last year and those who have not in a sample of 223 teachers working in educational institutions (elementary school level). Participants completed the Maslach Burnout Inventory (MBI) in its teachers' version, and a sociodemographic survey. A two -independent t test was carried out in order to see if both groups of teachers differed significantly in the values obtained for the MBI dimensions (emotional exhaustion, depersonalization, and lack of personal accomplishment). The results of the $t$ test showed significant differences between the groups of teachers, specifically in the dimensions of emotional exhaustion and depersonalization, showing higher values in the group of teachers who reported suffering from symptoms or health problems in the last 12 months.

Keywords: Primary School Teachers; Stress Related Diseases; Mental Health; Emotional Exhaustion; Depersonalization.

\section{Introducción}

La institución educativa es imprescindible para la vida en sociedad. La salud y la detección de posibles alteraciones psicofísicas en educandos y educadores es de primordial interés y ocupa un lugar importante entre los debates actuales por sus repercusiones en el proceso de enseñanza aprendizaje (Abuín \& Rivera, 2014).

El denominado síndrome de burnout (Maslach \& Jackson, 1981), o también conocido como síndrome de quemarse por el trabajo o desgaste psíquico laboral (Gil Monte \& Peiró Silla, 1997) fue desarrollado como un modelo teórico trifactorial por Maslach, y lo plantea como un síndrome que se observa especialmente en las profesiones de tipo asistencial (docentes, médicos, enfermeros, entre otras), caracterizado por tres manifestaciones principales: cansancio emocional, despersonalización y falta de realización personal (Blazer, 2010; Gutiérrez Ángel, 2019; Maslach \& Jackson, 1981; Maslach, 2009; Omdahl \& Fritz, 2006; Redó, 2009).

El desgaste psíquico profesional del docente puede generarse por una multiplicidad de factores, como son los cambios en las reformas educativas que exigen nuevas demandas, cambio en los roles, falta de autonomía, déficit en su reconocimiento a nivel social; la escasez de recursos apropiados; las interacciones con los alumnos, las cuales frecuentemente se encuentran caracterizadas por la indisciplina o la violencia escolar, como también la relación con las familias y autoridades educativas (Castillo Pérez, Moguel Luévano, Soto Nogeira Benítez Guadarrama \& 
Alvarado Tarango, 2017; Durán, Extremera, Montalbán \& Rey, 2005; Fernández, 2017; Scheuch, Haufe, \& Seibt, 2015).

Gil Monte y Peiró Silla plantean que se pueden diferenciar dos perspectivas en la conceptualización de este síndrome (1997):

a) La perspectiva clínica lo define como un estado al que llega el sujeto como consecuencia del estrés laboral, en esta dirección se encaminaron los primeros trabajos de Freudenberger (1974) y Fischer (1983)).

b) La perspectiva psicosocial lo plantea como un proceso que se desarrolla por la interacción de características del entorno laboral y personales, con manifestaciones bien diferenciadas en distintas etapas como se indica en los trabajos iniciales de Gil-Monte, Peiró y Valcárcel (1995), Golembiewski, Munzenrider y Carter (1983), Leiter (1988); Maslach y Jackson (1981), Schaufeli y Dierendonck (1993), entre otros.

Esta investigación se podría decir que se enfoca desde una perspectiva psicosocial, es decir, entendiendo el burnout como una derivación de eventos estresantes continuos e intensos vivenciados en el ámbito laboral, que disponen al individuo a padecerlo, sin posibilidad de afrontarlos adecuadamente y donde la interacción que el individuo mantiene con los diferentes condicionantes del trabajo son la clave para la aparición del burnout. En otras palabras, se lo considera como un proceso continuo que va surgiendo de una manera gradual y que se va instaurando en el individuo hasta inducir en éste los sentimientos propios del síndrome (Carlin, \& Garcés de los Fayos Ruiz, 2010; Zavala Zavala, 2008).

A lo largo del proceso se han observado diversos síntomas psicosomáticos, conductuales, emocionales, actitudinales e interpersonales que afectan al profesional y que se asocian al síndrome de burnout (Castillo Pérez, Moguel Luévano, Soto Nogeira Benítez Guadarrama \& Alvarado Tarango, 2017; Gil Monte \& Peiró Silla, 1997, Maslach \& Jackson, 1981; Menghi, 2016; Omdahl \& Fritz, 2006) (tabla 1).

Tabla 1.

Sintomas asociados al síndrome de burnout

\begin{tabular}{|c|c|c|c|c|}
\hline Emocionales & Actitudinales & Conductuales & Psicosomáticas & Interpersonales \\
\hline $\begin{array}{l}\text { El profesional } \\
\text { puede } \\
\text { experimentar: }\end{array}$ & $\begin{array}{l}\text { El profesional } \\
\text { puede tender a: }\end{array}$ & $\begin{array}{l}\text { El profesional } \\
\text { puede } \\
\text { comportase: }\end{array}$ & $\begin{array}{l}\text { El profesional puede } \\
\text { sentir: }\end{array}$ & $\begin{array}{l}\text { El profesional } \\
\text { puede tener: }\end{array}$ \\
\hline $\begin{array}{l}\text {-Sentimientos } \\
\text { de soledad, } \\
\text { de alienación, } \\
\text { de impotencia, } \\
\text { de omnipotencia } \\
\text {-Depresión } \\
\text {-Ansiedad }\end{array}$ & $\begin{array}{l}\text {-No expresar en } \\
\text { palabras } \\
\text {-Actitudes más } \\
\text { cínicas, apáticas, } \\
\text { hostiles, } \\
\text { desconfiadas }\end{array}$ & $\begin{array}{l}\text {-Agresivo } \\
\text {-Retraído } \\
\text {-Humor variado } \\
\text {-Enojado } \\
\text {-Irritable }\end{array}$ & $\begin{array}{l}\text {-Palpitaciones } \\
\text {-Problemas } \\
\text { cardiovasculares } \\
\text {-Hipertensión } \\
\text {-Crisis asmáticas } \\
\text {-Catarros frecuentes } \\
\text {-Tendencias a } \\
\text { infecciones, } \\
\text { enfermedades virales } \\
\text {-Aparición de alergias } \\
\text {-Dolores cervicales y de } \\
\text { espalda } \\
\text {-Fatiga } \\
\text {-Alteraciones } \\
\text { menstruales } \\
\text {-Úlceras gástricas } \\
\text {-Diarrea } \\
\text {-Migraña } \\
\text {-Desvelo }\end{array}$ & $\begin{array}{l}\text {-Conflictos } \\
\text { frecuentes con la } \\
\text { pareja } \\
\text {-Baja tolerancia } \\
\text {-Aislamiento } \\
\text {-Deterioro de las } \\
\text { relaciones }\end{array}$ \\
\hline
\end{tabular}

Nota: Adaptado de Gil Monte \& Peiró Silla (1997). 
Estos padecimientos o problemas de salud intervienen negativamente sobre la calidad de vida del individuo, afectando toda la red social del trabajador, debido a que las interacciones se hacen tensas y se tiende al aislamiento (Fernández, 2017; Mansilla Izquierdo, 2011). A medida que las personas se vuelven menos capaces de afrontar sus problemas laborales, se sienten más desgastadas psíquicamente en sus funciones por lo cual es probable que se retiren física y psicológicamente del trabajo, inviertan menos tiempo y energía en él, hagan sólo lo necesario y estén ausentes con mayor frecuencia, convirtiéndose en un funcionamiento de alto riesgo laboral (Menghi, 2016). Los maestros tienen deberes esenciales, contribuyen a la estabilidad de la sociedad y al desarrollo de las generaciones futuras (Scheuch, Haufe \& Seibt, 2015; Verdugo Maldonado \& Paucar Avila, 2018). Una alta calidad en el trabajo requiere tiempo, esfuerzo, compromiso y creatividad, y el individuo quemado no puede brindarlos (Kunter, et al. 2013).

Por tanto, entendiendo el síndrome como un proceso que surge de la interrelación de factores personales, laborales y sociales, es primordial el trabajo preventivo en salud mental y cuando los padecimientos están en curso la intervención temprana es crucial. La evidencia científica suele mostrar que existe cierta asociación entre el síndrome de burnout y el grado percibido de bienestar o salud (Ballester-Arnal, Gómez-Martínez, Gil-Juliá, Ferrándiz-Sellés, \& Collado-Boira, 2016; Esteras, Chorot \& Sandín, 2019) pero en nuestra región no se han hallado investigaciones que aporten conocimiento empírico, por consiguiente, este estudio podría ser un aporte relevante. Se tomarán como punto de referencia la percepción de síntomas o problemas de salud (mencionados por un grupo de docentes de educación primaria), siendo el objetivo principal conocer si existen diferencias significativas en los valores en las dimensiones del burnout en aquellos docentes de nivel primario que manifiestan síntomas o problemas de salud en los últimos 12 meses y los que no los manifiestan.

\section{Método}

\section{Participantes}

La muestra estuvo compuesta por 223 docentes, de sexo femenino y masculino (tabla 2), de instituciones educativas de gestiones públicas y privadas (tabla 3). Pertenecían a localidades de la provincia de Entre Ríos (Paraná, Concordia, Gualeguaychú, Nogoyá, María Grande) y Buenos Aires (tabla 3). La mayoría tenían entre 30 y 49 años de edad (70 \% de la muestra). Para la selección de los casos se empleó un muestreo no probabilístico, de tipo intencional. Los criterios de inclusión fueron que se encontraran ejerciendo frente al aula, en el nivel educativo primario y con más de 4 años de antigüedad en la docencia (tabla 3 ).

Tabla 2.

Distribución de edades por rango en función del sexo de los participantes

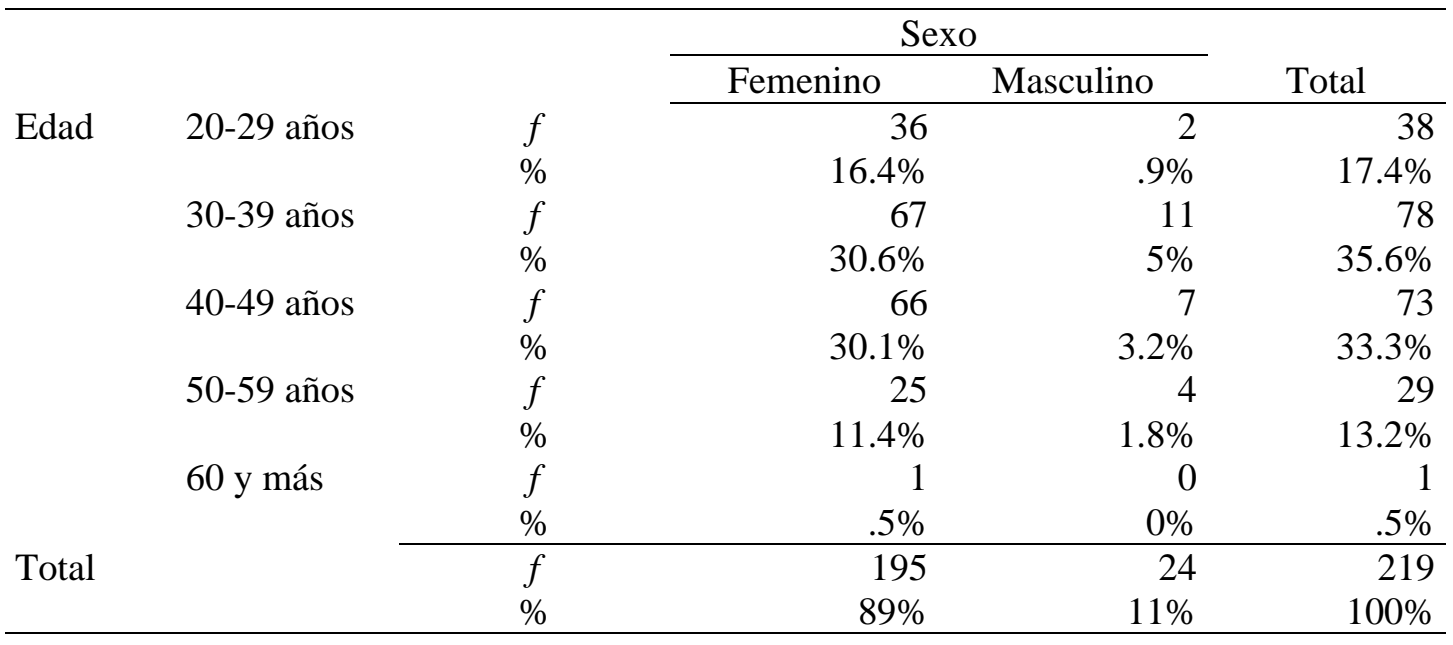


Tabla 3.

Frecuencias y porcentajes de variables sociodemográficas de los docentes

\begin{tabular}{rrr}
\hline Variables & $\boldsymbol{f}$ & $\%$ \\
\hline Centros Educativos & & \\
Públicos & 137 & 61.4 \\
Privados & 56 & 25.1 \\
Ambos & 27 & 12.1 \\
Antigüedad laboral & & \\
entre 5 y 9 años & 89 & 39.9 \\
entre 10 y 14 años & 54 & 24.2 \\
entre 15 y 19 años & 41 & 18.4 \\
entre 20 y más años & 34 & 15.2 \\
Localidades Gualeguaychú & 47 & \\
Concordia & 35 & 21.1 \\
Nogoyá & 50 & 15.7 \\
Paraná & 33 & 22.4 \\
María Grande & 23 & 14.8 \\
Buenos Aires & 35 & 10.3 \\
\end{tabular}

\section{Procedimiento}

Los participantes fueron contactados durante el segundo semestre del año 2016 y principios del año 2017 en instituciones educativas de gestiones públicas y privadas de las provincias de Entre Ríos y Buenos Aires. Se les informó en primer lugar los objetivos del estudio y la modalidad de participación. Los cuestionarios se entregaban a cada participante en forma de dossier (papel impreso), el cual contenía una nota de pedido de conformidad donde se solicitaba su firma (consentimiento informado), la encuesta para los datos sociodemográficos y a continuación el inventario de burnout. Los cuestionarios fueron autoadministrados. Para retirarlos se acordaba un día y horario. En total se repartieron 300 cuestionarios, de los cuales 223 fueron completados y devueltos en forma correcta. Todos los sujetos firmaron su consentimiento accediendo a participar de la investigación de manera voluntaria, sabiendo que los datos tendrían un tratamiento absolutamente confidencial y con fines de investigación.

\section{Instrumentos}

Encuesta sociodemográfica. Se construyó esta encuesta ad hoc para recabar información sobre: el sexo, la edad, el grado en el que se desempeña, el tipo de institución (pública-privada), la antiguiedad profesional. Para recabar datos específicos acerca de los síntomas o problemas de salud se indagó mediante las siguientes preguntas:

a) "En los últimos 12 meses ¿ha experimentado algunos de estos síntomas o problemas de salud de manera recurrente? Marque o especifique si no aparece la opción.

1. Resfríos/ Gripe /Contracturas musculares/ Alergias/ Ninguno/ Otros:

2 .

b) ¿Debió sacar licencia a causa de dificultades en su salud?: No / Si. En caso de la que la respuesta sea $\mathrm{Si}$ ¿Cuál fue el diagnóstico?:

3.

4. En la construcción de la pregunta semi estructurada a), se optó por denominar síntomas o problemas de salud para ampliar las posibilidades y que pudieran mencionar afecciones en su salud que se les hayan presentado en el último año, sea cual fuera la forma en que lo perciben y experimentan debido a que cada sujeto lo puede reconocer de modo diferente, 
como ser: enfermedad, entendiéndolo como algo crónico, otros como problemas de salud momentáneos y otros como síntomas de algo que no tiene diagnóstico.

5.

Inventario Burnout (MBI; Maslach \& Jackson, 1981). Mide los sentimientos y actitudes que tiene el profesional hacia su trabajo. En esta investigación se aplicó la versión para educadores. Consta de 22 afirmaciones, agrupadas en tres dimensiones: (a) cansancio emocional, (b) despersonalización y (c) baja realización personal. Las frecuencias de las afirmaciones se estiman en una escala tipo Likert de siete opciones: "nunca"; "casi nunca"; "pocas veces"; "a veces"; "bastante"; "muchas veces"; "todos los días". La escala en su versión en español tiene una consistencia interna y una confiabilidad medida a través del alpha de Cronbach de .90 en CE; .79 en DP y .71 en RP (Seisdedos, 1997). La confiabilidad de esta escala en la muestra de estudio medida a través del alfa de Cronbach es de .72 para la escala completa y para las dimensiones los valores fueron de .90 en CE, de .57 en DP y de .78 en RP. En general, en muestras locales, los valores de alpha han mostrado ser aceptables, aunque bastante más bajos para la dimensión de Despersonalización. Por ejemplo, Cabrera (2003) utilizó el instrumento con una muestra de la provincia de Entre Ríos, encontrando un coeficiente de fiabilidad de .84 para la subescala CE; .42 para DP y de .72 para RP. Por su parte, Marucco, Flamenco y Ragazzoli (2009), en su estudio con población bonaerense, hallaron un coeficiente de fiabilidad de .80 para RP; .55 para DP; y .90 para CE. Menghi y Oros (2014) en su investigación en una muestra de docentes argentinos de las provincias de Entre Ríos y Buenos Aires encontraron un alpha de Cronbach de .85 para Cansancio Emocional; .55 para Despersonalización y .83 para Realización Personal, y además para complementar los análisis psicométricos de la escala realizaron un análisis factorial exploratorio encontrando tres claras dimensiones que replican la estructura factorial propuesta por los autores Maslach y Jackson (Método Componentes principales, rotación Varimax. $\mathrm{KMO}=.884$; Chi cuadrado de Bartlett $(231)=2211.285 ; \mathrm{p}=.000$. Var. Explicada $=48.95 \%)$. A pesar de que la consistencia interna de la escala DP ha resultado ser relativamente baja, reflejando similarmente los valores obtenidos con otras muestras nacionales, estadounidenses y españolas, el MBI constituye un instrumento de gran utilidad que, dada su difusión, permite cotejar resultados con otros estudios (Gil-Monte \& Peiró, 1997).

\section{Resultados}

Para analizar los grupos de docentes que manifestaron síntomas o problemas de salud en los últimos 12 meses y los que no manifestaron, se redujo el número de sujetos que manifestaron padecer síntomas o problemas de salud mediante el criterio de selección aleatoria de casos de la base de datos, debido a que los grupos no eran equivalentes en número (tabla 4). Por lo tanto, los análisis de diferencia de grupo se llevaron a cabo con 54 docentes, 27 en cada grupo (tabla 5).

\section{Tabla 4.}

Composición de la muestra de docentes en función de la presencia o ausencia de síntomas o problemas de salud en los últimos 12 meses

\begin{tabular}{lcc}
\hline Docentes & $f$ & $\%$ \\
\hline $\begin{array}{l}\text { Han presentado síntomas/ p. } \\
\text { salud en los últimos 12 } \\
\text { meses }\end{array}$ & 196 & $88 \%$ \\
\hline $\begin{array}{l}\text { No han presentado síntomas/ } \\
\text { p. salud en los últimos 12 } \\
\text { meses }\end{array}$ & 27 & $12 \%$ \\
\hline \multicolumn{1}{c}{$\mathrm{N}$} & 223 & $100 \%$ \\
\hline
\end{tabular}

Nota: síntomas/p. salud: síntomas o problemas de salud 
Tabla 5.

Características de la muestra en función de los dos grupos

\begin{tabular}{|c|c|c|c|c|}
\hline \multirow[b]{2}{*}{ Variables } & \multicolumn{2}{|c|}{$\begin{array}{l}\text { Han presentado síntomas/ p. } \\
\text { salud en los últimos } 12 \text { meses }\end{array}$} & \multicolumn{2}{|c|}{$\begin{array}{l}\text { No han presentado síntomas/ } \\
\text { p. salud en los últimos } 12 \\
\text { meses }\end{array}$} \\
\hline & $f$ & $\%$ & $f$ & $\%$ \\
\hline \multicolumn{5}{|l|}{ Sexo } \\
\hline Femenino & 24 & 45.3 & 24 & 45.3 \\
\hline Masculino & 3 & 5.7 & 3 & 5.7 \\
\hline \multicolumn{5}{|l|}{ Antigüiedad laboral } \\
\hline entre 5 y 9 años & 6 & 11.5 & 13 & 25 \\
\hline entre 10 y 14 años & 9 & 17.3 & 4 & 7.7 \\
\hline entre 15 y 19 años & 6 & 11.5 & 4 & 7.7 \\
\hline entre 20 y más años & 6 & 11.5 & 6 & 11.5 \\
\hline
\end{tabular}

Nota: síntomas/p. salud: síntomas o problemas de salud

Como se puede apreciar en la tabla 6, se han encontrado diferencias significativas en los valores de la dimensión cansancio emocional y en la dimensión despersonalización entre los grupos que han experimentado síntomas o problemas de salud y los que no, siendo mayor el nivel de cansancio emocional y el nivel de despersonalización en el primer grupo de docentes.

Tabla 6.

Prueba t para muestras independientes en grupos de docentes de nivel primario

\begin{tabular}{|c|c|c|c|c|c|c|c|}
\hline & \multicolumn{2}{|c|}{$\begin{array}{l}\text { No han presentado } \\
\text { síntomas /p. salud en } \\
\text { los últimos } 12 \text { meses } \\
(\mathrm{n}=27)\end{array}$} & \multicolumn{2}{|c|}{$\begin{array}{c}\text { Si han presentado } \\
\text { síntomas/ p. salud en } \\
\text { los últimos } 12 \text { meses } \\
(n=27)\end{array}$} & \multicolumn{2}{|c|}{ Valores estadísticos } & \multirow[t]{2}{*}{$95 \% \mathrm{IC}$} \\
\hline & $M$ & $D E$ & $M$ & $D E$ & $t$ & $p$ & \\
\hline $\mathrm{CE}$ & 1.56 & .91 & 2.16 & 1.34 & -1.94 & .05 & {$[-1.23, .02]$} \\
\hline DP & .63 & .73 & 1.05 & .75 & -2.13 & .03 & {$[-.83,-.02]$} \\
\hline
\end{tabular}

Nota: CE: cansancio emocional, DP: despersonalización

Además, en este trabajo se identificaron los principales síntomas o problemas de salud autoinformados por los docentes en la encuesta ad hoc ante la pregunta: "En los últimos 12 meses ¿ha experimentado algunos de estos síntomas o problemas de salud de manera recurrente? Marque o especifique si no aparece la opción.

Resfríos/ Gripe /Contracturas musculares/ Alergias/ Ninguno/ Otros:

El 88\% ( $\mathrm{n}=196)$ de los docentes encuestados mencionaron experimentar algunos de los siguientes síntomas o problemas de salud: Arritmias, Gripe, Bronquitis, Cervicalgia, Hipertensión, Hipotensión, Daño en las cuerdas vocales, Crisis asmáticas, Hipoacusia súbita, Tendinitis, Esguinces, Migrañas severas, Neumonía, Anemia, Descomposturas, Gastritis, Infecciones, Infecciones urinarias, Trastornos del sueño, Mareos, Disfonía, Problemas visuales diversos, entre otros.

Con la finalidad de observar los síntomas o problemas de salud más recurrentes en esta muestra, en la tabla 7 se describen los mencionados por los maestros con mayor frecuencia. 
Tabla 7.

Problemas de salud más frecuentes de los docentes de nivel educativo primario

\begin{tabular}{lll}
\hline & $f$ & $\%(n=196)$ \\
\hline Estrés & 19 & 10 \\
Hipertensión & 17 & 9 \\
Depresión; Gripe & 15 & 8 \\
Faringitis; Neumonía & 13 & 7 \\
Ansiedad; Cervicalgia; Anemia; Bronquitis & 12 & 6 \\
\hline
\end{tabular}

Nota: se describen solo los que tuvieron la misma denominación por los sujetos y que tuvieron una frecuencia superior a 3 .

\section{Discusión}

La educación es una de las profesiones de carácter asistencial (como la medicina, la enfermería, entre otros) con mayores riesgos psicosociales. La Organización Internacional del Trabajo ya por los años ochenta suministró información acerca del trabajo docente como una de las labores de riesgo de sufrir agotamiento físico y mental, y subrayaba que el problema del estrés crónico era una de las principales causas de abandono de dicha labor (1984).

El desgaste psíquico profesional en el docente, puede generarse por una multiplicidad de factores como son los cambios en las reformas educativas que exigen nuevas demandas, cambio en los roles, falta de autonomía, déficit en su reconocimiento a nivel social; la escasez de recursos apropiados; las interacciones con los alumnos, las cuales frecuentemente se encuentran caracterizadas por la indisciplina o la violencia escolar, como también la relación con las familias y autoridades educativas (Castillo Pérez, Moguel Luévano, Soto Nogeira Benítez Guadarrama \& Alvarado Tarango, 2017; Durán, Extremera, Montalbán \& Rey, 2005; Fernández, 2017; Scheuch, Haufe, \& Seibt, 2015). En suma, la interacción que el individuo mantiene con los diferentes condicionantes del trabajo y sus escasos recursos personales para afrontarlos crean el camino propicio para el desarrollo del síndrome (Carlin, \& Garcés de los Fayos Ruiz, 2010; Menghi, 2016; Zavala Zavala, 2008).

Mediante este estudio se han hallado indicios de este proceso de desgaste psíquico planteado por la teoría y sus investigadores referentes (Maslach \& Jackson, 1981; Gil Monte \& Peiró Silla, 1997), encontrándose que el cansancio emocional y el sentimiento de despersonalización estarían acompañados de diversos padecimientos, ya que quienes obtuvieron los valores más elevados en estas dimensiones son los que dicen haber experimentado síntomas o problemas de salud en el último tiempo. Según Juarez García et al. el agotamiento y la despersonalización son los constructos clave del síndrome y están asociados en mayor medida a diversas consecuencias laborales (Juárez García, Idrovo, Camacho Ávila, Placencia Reyes, 2014). Por otro lado, la investigación de Esteras, Chorot y Sandín (2019) examinó la asociación del síndrome de burnout y la sintomatología somática y psicopatológica en docentes de preescolar, primaria y bachillerato, encontrando que el agotamiento emocional correlaciona de forma significativa con la sintomatología somática, siendo las correlaciones más elevadas las que conciernen a los síntomas de tipo musculo-esquelético, inmunológico general, cardiovascular, neurosensorial y gastrointestinal, en las dimensiones despersonalización y realización personal las correlaciones fueron más bajas. Estos autores postulan que el agotamiento emocional podría generar las condiciones para el desarrollo de enfermedades físicas.

Los resultados de Esteras, Chorot y Sandín (2019) son acordes a las investigaciones pioneras acerca del burnout, donde el síndrome aparece asociado a trastornos físicos diversos, incluidos los distintos tipos de dolor y molestias físicas, y a las alteraciones de índole cardiovascular, respiratorio, inmunológico e infeccioso (Maslach \& Jackson, 1981; Gil Monte \& Peiró Silla, 1997). En este estudio, aunque solo con la finalidad de observar presencia o ausencia, se indagaron los síntomas o problemas de salud percibidos dando lugar a expresiones libres de 
parte de los docentes ante esta pregunta, surgiendo variadas respuestas y entre las más frecuentes mencionaron: el estrés, hipertensión arterial, depresión, gripe, faringitis, neumonía, ansiedad, cervicalgia, anemia, bronquitis, todos ellos, síntomas que podrían agruparse según Gil Monte y Peiró Silla (1997) en trastornos somáticos, psicológicos y emocionales.

Cabe mencionar algunas de las limitaciones en la investigación presentada, como el tipo de muestreo utilizado, ya que no es posible una generalización representativa de los resultados a la población en general sino una mera aproximación a los fenómenos evaluados en este grupo de docentes. Otra limitación para mencionar es que la recolección de los datos se hizo a través de cuestionarios autoadministrados, lo que restringe en alguna medida la comprensión en profundidad del fenómeno. En suma, el diseño metodológico seleccionado permite una visión parcial del fenómeno.

Para finalizar, se recomienda a futuras investigaciones, aumentar el tamaño de la muestra y profundizar en la investigación de estas variables estudiando las relaciones entre ellas en pos de contar con mayor evidencia empírica para la intervención temprana ante los primeros indicios del síndrome de burnout y sus complejas consecuencias que afectan al docente y a todo el sistema.

\section{Referencias}

Abuín M. R. \& Rivera L. (2014). La medición de síntomas psicológicos y psicosomáticos: el Listado de Síntomas Breve (LSB-50). Clínica y Salud 25, 131-141. doi: http://dx.doi.org/10.1016/j.clysa.2014.06.001

Blazer, C. (2010). Teacher Burnout. Information Capsule. ResearchServices, Miami-Dade County PublicSchools. Recuperado de http://eric.ed.gov/?id=ED536515

Cabrera, M. (2003). Estudio sobre el síndrome de burnout en funcionarios policiales y su relación con el locus de control laboral y características demográficas (Tesis de Licenciatura en Psicología, inédita). Universidad Adventista del Plata, Libertador San Martín, Entre Ríos, Argentina.

Carlin, M., \& Garcés de los Fayos Ruiz, E. J. (2010). El síndrome de burnout: Evolución histórica desde el contexto laboral al ámbito deportivo. Anales de psicología, 26(1), 169-180.

Castillo Pérez, V., Moguel Luévano, M., Soto Nogeira, L., Benítez Guadarrama, J., \& Alvarado Tarango, L., (2017). Síntomas y Efectos del Síndrome del "Burnout", una Revisión Sistemática. Academia Journals, 6(1), 79-85.

Durán, A., Extremera, N., Montalbán, F., \& Rey, L. (2005). Engagement y Burnout en el ámbito docente: Análisis de sus relaciones con la satisfacción laboral y vital en una muestra de profesores. Revista de Psicología del Trabajo y las Organizaciones, 21, 145 - 158.

Esteras, J., Chorot, P., \& Sandín, B. (2019). Sintomatología física y mental asociada al síndrome de burnout en los profesionales de la enseñanza. Revista de Psicopatología y Psicología Clínica, 24 (1), 29-37. Recuperado de http://revistas.uned.es/index.php/rppc

Fernández, M.P. (2017). Relación entre desgaste ocupacional y manifestaciones psicosomáticas en una muestra de docentes colombianos de la ciudad de Santa Marta. ArchMed (Manizales); 17(1),78-90. Recuperado de http://revistasum.umanizales.edu.co/ojs/index.php/archivosmedicina/article/view/1910/2 228

Fischer, H. J. (1983). A psychoanalytic view of burnout. En B. A. Farber (ed.), Stress and burnout in the human services professions (1985, 2da ed., pp. 40-45). New York: Pergamon Press.

Freudenberger, H. J. (1974). Staff burnout. Journal of Social Issues, 30(1), 159-165. Doi: https://doi.org/10.1111/j.1540-4560.1974.tb00706.x

Gil Monte, P.R., \& Peiró Silla, J. M. (1997). Desgaste psíquico en el trabajo: El síndrome de quemarse. Madrid: Editorial Síntesis.

Gil-Monte, P. R., Peiró, J. M. \& Valcárcel, P. (1995). A causal model of burnout process development: An alternative to Golembiewski and Le-iter Models. Trabajo presentado en el VII Congreso Europeo sobre Trabajo y Psicología Organizacional. Gyor: Hungría 
Golembiewski, R. T., Munzenrider, R. \& Carter, D. (1983). Phases of pro-gressive burnout and their work site co-variants: Critical issues in OD research and praxis. Journal of Applied Behaviour Science, 19(4), 461- 481. doi: https://doi.org/10.1177/002188638301900408

Gutiérrez Ángel, N., (2019). Análisis de las publicaciones sobre el síndrome de burnout en el profesorado español en los últimos años (2007-2017). Revista Boletín Redipe, 8 (1), 27 32. Recuperado de https://revista.redipe.org/index.php/1/article/view/678/643

Juárez García A., Idrovo A., Camacho Ávila A., Placencia Reyes O. (2014). Síndrome de burnout en población mexicana: Una revisión sistemática. Salud Mental, 159-176. Recuperado de http://www.scielo.org.mx/pdf/sm/v37n2/v37n2a10.pdf

Leiter, M. P. (1988). Burnout as a function of communication patterns. Group and Organization Studies, 13(1), 111-128. Recuperado de https://elibrary.ru/item.asp?id=11249469

Mansilla Izquierdo, F. (2011). Adicción al trabajo en la administración pública. Psicología.com, 15. Recuperado de: http://www.psiquiatria.com

Marucco, M.A., Flamenco, E., \& Ragazzoli, P.N. (2009). Estudio para evaluar el Síndrome de Quemarse por el Trabajo y la Calidad de Vida Laboral en docentes de Educación Primaria Básica. Superintendencia de Riesgos del Trabajo. Recuperado de http://www.infoleg.gov.ar/basehome/actos_gobierno/actosdegobierno19-10-2009-4.htm

Maslach, C. (2009). Comprendiendo el Burnout. Ciencia \& Trabajo, 11(32), 31-43.

Maslach, C., \& Jackson, S. E. (1981). The measurement of experienced burnout. Journal of Occupational Behaviour, 99-113. doi: https://doi.org/10.1002/job.4030020205

Menghi, M.S. (2016). Recursos psicosociales y manejo del estrés en docentes. (Tesis de doctorado en Psicología, inédita). Facultad de Psicología y Psicopedagogía, Universidad Católica Argentina, Buenos Aires.

Menghi, M.S., \& Oros, L. B. (2014). Satisfacción laboral y Síndrome de Burnout en docentes de nivel primario. Revista de Psicología, 10(20), 47-59. Recuperado de http://hdl.handle.net/11336/9968

Omdahl, B.L., \& Fritz, J.M.H. (2006). Coping with Problematic Relationships in the Workplace: Strategies that Reduce Burnout. Paper presented at the annual meeting of the International Communication Association, Dresden Germany.

Organización Internacional del Trabajo/Organización Mundial de la Salud. (1984). Informe del Comité Mixto OIT/OMS sobre medicina del trabajo. Factores psicosociales en el trabajo: naturaleza, incidencia y prevención. Ginebra, Suiza. Recuperado de http://staging.ilo.org/public/libdoc/ilo/1986/86B09_301_span.pdf

Redó, N. A. (2009). El Síndrome de Burnout en los docentes. Electronic Journal of Research in Education Psychology, 7(18), 829-848. Recuperado de http://www.investigacionpsicopedagogica.org/revista/articulos/18/espannol/Art_18_303.pdf

Schaufeli, W. B. \& Dierendonck, D. (1993). The construct validity of two burnout measures. Journal of Occupational Behaviour, 14, 631-647. Recuperado de https://www.jstor.org/stable/2488226?seq=1\#page_scan_tab_contents

Scheuch, K., Haufe, E., \& Seibt, R. (2015). Salud de los docentes. Deutsches Arzteblatt international, 112(20), 347-356. doi: http://dx.doi.org/10.3238 /arztebl.2015.0347

Seisdedos, N. (1997). Manual MBI, Inventario Burnout de Maslach. Madrid: TEA.

Zavala Zavala, J. (2008). Estrés y burnout docente: conceptos, causas y efectos. Educación 17(32), 67-86. Recuperado http://revistas.pucp.edu.pe/index.php/educacion/article/view/1802 